\title{
An Application of Potential Estimates to A Priori Bounds for Elliptic Equations
}

\author{
Farman Mamedov, ${ }^{1}$ Sara Monsurrò, ${ }^{2}$ and Maria Transirico ${ }^{2}$ \\ ${ }^{1}$ Mathematics and Mechanics Institute, Azerbaijan National Academy of Sciences, 10 Istiglaliyyat Street, Az1001, Baku, Azerbaijan \\ ${ }^{2}$ Dipartimento di Matematica, Università di Salerno, Via Giovanni Paolo II 132, 84084 Fisciano, Italy
}

Correspondence should be addressed to Sara Monsurrò; smonsurro@unisa.it

Received 15 December 2015; Accepted 3 February 2016

Academic Editor: Julio D. Rossi

Copyright (c) 2016 Farman Mamedov et al. This is an open access article distributed under the Creative Commons Attribution License, which permits unrestricted use, distribution, and reproduction in any medium, provided the original work is properly cited.

A potential estimate type approach is used in order to obtain some a priori bounds for the solutions of certain classes of Dirichlet problems associated with nondivergence structure elliptic equations.

\section{Introduction}

In this paper we are concerned with some aspects related to the study of the strong solvability of the classical Dirichlet problem

$$
\begin{aligned}
L u & =-f, \quad f \in L^{p}(\Omega), \\
\left.u\right|_{\partial \Omega} & =0
\end{aligned}
$$

in the space $W^{2, p}(\Omega), p>1$, where $\Omega$ is a sufficiently regular bounded open subset of $\mathbb{R}^{n}, n>2$, and $L$ is the second-order linear differential operator in nondivergence form

$$
L=\sum_{i, j=1}^{n} a_{i j}(x) \frac{\partial^{2}}{\partial x_{i} \partial x_{j}}
$$

As evidenced by a renewed example by C. Pucci (quoted by Talenti in [1]), for $n>2$, the boundedness and ellipticity of the $a_{i j}$ are not enough to derive the strong solvability of problem (1). There are two ways to overcome this difficulty: the first approach consists in imposing suitable regularity of the $a_{i j}$, while the second one assumes that the coefficients do not scatter too much, satisfying conditions stronger than the uniform ellipticity (see, e.g., [2] for a wider survey on this subject).

Concerning the first way, classical results (see, e.g., [3]) ensure that, for sufficiently regular sets $\Omega$ and for coefficients $a_{i j} \in C^{0}(\bar{\Omega}), i, j=1, \ldots, n$, one obtains the unique strong solvability of (1) together with the bound

$$
\|u\|_{W^{2, p}(\Omega)} \leq C\|f\|_{L^{p}(\Omega)} .
$$

The embedding theorems then give

$$
\|u\|_{L^{n p /(n-2 p)}(\Omega)} \leq C\|f\|_{L^{p}(\Omega)},
$$

for $p<n / 2$. Let us point out that the constant $C$ in (4) is independent of $u$ but depends on the required regularity of the coefficients.

Following the second approach, Talenti obtains existence and uniqueness results for problem (1), for $p=2$, considering, in [1], coefficients satisfying the so-called Cordes condition:

$$
\frac{\sum_{i, j=1}^{n} a_{i j}^{2}(x)}{\left(\sum_{i=1}^{n} a_{i i}(x)\right)^{2}} \leq \frac{1}{n-1}-\delta, \quad \delta>0 .
$$

Condition (5) allows him to prove the estimate

$$
\|u\|_{W^{2,2}(\Omega)} \leq C\|f\|_{L^{2}(\Omega)} .
$$

Later on, Campanato, in [4], extended these results to values of $p$ sufficiently close to 2 .

Taking into account these results, in the recent paper [5], we assume that the coefficients $a_{i j} \in C^{0}(\bar{\Omega}), i, j=1, \ldots, n$, 
are symmetric bounded measurable functions satisfying the following new hypothesis:

$$
l=\underset{x \in \Omega, \xi \in \mathbb{R}^{n},|\xi|=1}{\operatorname{essinf}} \frac{\sum_{i=1}^{n} a_{i i}(x)}{\sum_{i, j=1}^{n} a_{i j}(x) \xi_{i} \xi_{j}}>2 .
$$

Hypothesis (7) generalizes the classical Cordes condition, since (see Remark 3 ), for $n>5$, the class of operators satisfying (7) is wider than the one verifying Cordes assumption.

In [5] we prove estimate (4), showing that, for the range of exponent $n / l<p<n / 2$, the constant $C$ is independent of the regularity of the coefficients. This can be done by assuming that $f$ has an extension, belonging to certain generalized Sobolev spaces $L_{\nu, p}^{\alpha}\left(\mathbb{R}^{n}\right)$ (see Section 2 ) verifying quite restrictive conditions essentially due to the lack in literature of opportune extension results in the involved functional spaces. We point out that obtaining an estimate with a constant independent of the regularity of the coefficients is an important feature of the bound, since it can allow weakening of the hypotheses on the regularity of the $a_{i j}$.

The goal of the present work is to achieve an a priori bound for the solution of problem (1) in the same framework of [5], but dropping the restrictive conditions on the datum $f$, required therein.

Our main result, obtained assuming that $f$ is the restriction to $\Omega$ of a function $\bar{f}$ belonging to $L_{v, p}^{\alpha}\left(\mathbb{R}^{n}\right)$ (roughly speaking, $f \in L^{p}(\Omega)$ is the restriction to $\Omega$ of the Riesz potential $I^{\alpha}(g)$ of a function $g \in L^{\nu}\left(\mathbb{R}^{n}\right)$, with $\left.\alpha=n / \nu-n / p\right)$, consists in the following estimate:

$$
\|u\|_{L^{n p /(n-2 p)}(\Omega)} \leq C\|\bar{f}\|_{L_{\nu, p}^{\alpha}\left(\mathbb{R}^{n}\right)}
$$

for $n / l<p<n / 2$, where the constant $C$ is again independent of the regularity of the coefficients.

The main tools in achieving (8) are certain potential type estimates previously obtained for the solutions of problems of the same kind of (1), but with more regular datum. We refer to estimates (35) in Step 1 and (52) in Step 2 of Theorem 5. We remark that hypothesis (7) is the key point in the proof of Lemma 4, which allows showing the abovementioned potential type bounds.

\section{A Class of Generalized Sobolev Spaces}

This section is devoted to the definition and the main properties of a class of generalized Sobolev spaces where the datum $f$ will be taken (see also [5]).

To our aim, we recall the main features of Riesz potentials and hypersingular integrals focusing just on some specific aspects, required to our needs. For wider and deeper surveys we refer the reader, for instance, to [6-9].

Let $0<\alpha<n$ and $1 \leq v<+\infty$. It is known that if $g \in L^{v}\left(\mathbb{R}^{n}\right)$ then the integral

$$
\left(I^{\alpha} g\right)(x)=\int_{\mathbb{R}^{n}} \frac{g(y)}{|x-y|^{n-\alpha}} d y, \quad x \in \mathbb{R}^{n},
$$

converges absolutely for almost every $x$.
The function $I^{\alpha} g$ in (9) defines, up to a positive multiplicative constant depending on $\alpha$, the Riesz potential of $g$.

For $0<\alpha<n$ and $1 \leq v<+\infty$, it is therefore possible to consider the space

$$
I^{\alpha}\left(L^{\nu}\right)=\left\{f: f=I^{\alpha}(g), g \in L^{\nu}\left(\mathbb{R}^{n}\right)\right\}
$$

that is, a Banach space, with respect to the norm

$$
\|f\|_{I^{\alpha}\left(L^{\nu}\right)}=\|g\|_{L^{\nu}\left(\mathbb{R}^{n}\right)} .
$$

If, in addition, $1<v<n / \alpha$, one has the following strict inclusion:

$$
I^{\alpha}\left(L^{\nu}\right) \subset L^{q}\left(\mathbb{R}^{n}\right), \quad \text { with } q=\frac{n v}{n-\alpha \nu},
$$

and moreover

$$
\left\|I^{\alpha} g\right\|_{L^{q}\left(\mathbb{R}^{n}\right)} \leq c\|g\|_{L^{y}\left(\mathbb{R}^{n}\right)}
$$

Now, let $0<\alpha<n, 1<\nu<n / \alpha$, and $q=n v /(n-\alpha v)$. A characterization of hypersingular integrals in terms of Riesz potentials (see [7]) allows us to define the space $L_{v, q}^{\alpha}\left(\mathbb{R}^{n}\right)$ of hypersingular integrals as

$$
\begin{aligned}
& L_{v, q}^{\alpha}\left(\mathbb{R}^{n}\right) \\
& \quad=\left\{f \in L^{q}\left(\mathbb{R}^{n}\right): f=I^{\alpha}(g) \text { with } g \text { in } L^{\nu}\left(\mathbb{R}^{n}\right)\right\} .
\end{aligned}
$$

The function $g$ in (14) is called the Riesz derivative of $f$; it is denoted by $\mathbb{D}^{\alpha} f$ and can be interpreted as an inverse of the Riesz potential.

For $0<\alpha<n, 1<\nu<n / \alpha$, and $q=n v /(n-\alpha \nu)$, the space $L_{\nu, q}^{\alpha}\left(\mathbb{R}^{n}\right)$ can be therefore rewritten as

$$
L_{v, q}^{\alpha}\left(\mathbb{R}^{n}\right)=\left\{f \in L^{q}\left(\mathbb{R}^{n}\right): \mathbb{D}^{\alpha} f \text { in } L^{\nu}\left(\mathbb{R}^{n}\right)\right\}
$$

and it is a Banach space endowed with the norm

$$
\|f\|_{L_{\gamma, q}^{\alpha}\left(\mathbb{R}^{n}\right)}=\|f\|_{L^{q}\left(\mathbb{R}^{n}\right)}+\left\|\mathbb{D}^{\alpha} f\right\|_{L^{\nu}\left(\mathbb{R}^{n}\right)}
$$

From definition (15) it appears to be clear that $L_{v, q}^{\alpha}\left(\mathbb{R}^{n}\right)$ generalizes the notion of Sobolev space to the fractional case.

It is known that $C_{0}^{\infty}\left(\mathbb{R}^{n}\right)$ is dense in $L_{v, q}^{\alpha}\left(\mathbb{R}^{n}\right)$, where, as usual, $C_{0}^{\infty}\left(\mathbb{R}^{n}\right)$ stands for the class of all $C^{\infty}$ functions on $\mathbb{R}^{n}$ with compact support.

Definition 1. Let $\Omega$ be an open subset of $\mathbb{R}^{n}, n>2$. For $0<$ $\alpha<n, 1<\nu<n / \alpha$, and $q=n \nu /(n-\alpha \nu)$, we define $L_{\nu, q}^{\alpha}(\Omega)$ as the set of restrictions to $\Omega$ of functions in $L_{v, q}^{\alpha}\left(\mathbb{R}^{n}\right)$. Namely, $f \in L_{v, q}^{\alpha}(\Omega)$ if there exists a function $\bar{f} \in L_{v, q}^{\alpha}\left(\mathbb{R}^{n}\right)$ such that $\bar{f}=f$ in $\Omega$. norm

The space $L_{v, q}^{\alpha}(\Omega)$ is a Banach space endowed with the

$$
\|f\|_{L_{\nu, q}^{\alpha}(\Omega)}=\inf \left\{\|\bar{f}\|_{L_{\nu, q}^{\alpha}\left(\mathbb{R}^{n}\right)} \mid \bar{f}_{\mid \Omega}=f\right\} .
$$




\section{Main Result}

Let $\Omega$ be an open subset of $\mathbb{R}^{n}, n>2$. We want to prove some a priori estimates for strong solutions of the following Dirichlet problem:

$$
\begin{gathered}
u \in W^{2, p}(\Omega) \cap \stackrel{\circ}{W}^{1, p}(\Omega), \\
L u=-f, \quad f \in L^{p}(\Omega),
\end{gathered}
$$

$p>1$.

Here $L$ is a second-order linear differential operator in nondivergence form

$$
L=\sum_{i, j=1}^{n} a_{i j}(x) \frac{\partial^{2}}{\partial x_{i} \partial x_{j}},
$$

with coefficients $a_{i j}$ that are symmetric bounded measurable functions satisfying the uniform ellipticity condition; therefore, there exists a constant $\mu \in(0,1)$ such that

$$
\mu|\xi|^{2} \leq \sum_{i, j=1}^{n} a_{i j}(x) \xi_{i} \xi_{j} \leq \mu^{-1}|\xi|^{2}
$$

for almost every $x \in \Omega$ and for any $\xi \in \mathbb{R}^{n}$.

In the sequel we assume that

$$
a_{i j} \in C^{0}(\bar{\Omega}), \quad i, j=1, \ldots, n,
$$

and set

$$
l=\underset{x \in \Omega, \xi \in \mathbb{R}^{n},|\xi|=1}{\operatorname{ess} \inf _{i, j=1}} \frac{\sum_{i=1}^{n} a_{i i}(x)}{\sum_{i j}^{n}(x) \xi_{i} \xi_{j}},
$$

and we suppose that

$$
l>2
$$

Condition (23) is equivalent to

$$
l=\inf _{x \in \Omega} \frac{\sum_{i=1}^{n} \lambda_{i}(x)}{\max _{1 \leq i \leq n}\left\{\lambda_{i}(x)\right\}}>2,
$$

where $\lambda_{i}, i=1, \ldots, n$, are the eigenvalues of the matrix $\left\|a_{i j}\right\|$. Remark 2. By the definition of $l$ it easily follows that $1<l<n$.

Remark 3. For $n>5$ the class of operators satisfying the assumption (23) is wider than the one verifying the Cordes condition. Indeed the Cordes assumption reads as

$$
\frac{\sum_{i, j=1}^{n} a_{i j}^{2}(x)}{\left(\sum_{i=1}^{n} a_{i i}(x)\right)^{2}} \leq \frac{1}{n-1}-\delta, \quad \delta>0 \text {, a.e. in } \Omega,
$$

or equivalently

$$
\frac{\sum_{i=1}^{n} \lambda_{i}^{2}(x)}{\left(\sum_{i=1}^{n} \lambda_{i}(x)\right)^{2}} \leq \frac{1}{n-1}-\delta, \quad \delta>0, \text { a.e. in } \Omega \text {. }
$$

Thus, if (26) is satisfied, by (26) and by the definition of $l$, it follows that $l>\sqrt{n-1}$. This gives (24) for $n>5$.
Let us now state the following lemma, proved in [5], that is an essential tool in the proof of our main result.

Lemma 4. Let $L$ be the operator defined in (19) satisfying (20) and (23). If $n>2,0<s<l-2$, and $g$ is a nonnegative function in $L^{v}\left(\mathbb{R}^{n}\right)$, for some fixed $1<v<n /(n-s)$, then for sufficiently small $\varepsilon>0$ the potential

$$
W^{\varepsilon}(x)=\int_{\mathbb{R}^{n}} \frac{g(y)}{\left(|x-y|^{2}+\varepsilon^{2}\right)^{s / 2}} d y, \quad x \in \Omega,
$$

is such that

$$
\begin{aligned}
& L W^{\varepsilon}(x) \\
& \quad \leq-\mu s(l-s-2) \int_{\mathbb{R}^{n}} \frac{g(y)}{\left(|x-y|^{2}+\varepsilon^{2}\right)^{(s+2) / 2}} d y \leq 0,
\end{aligned}
$$

for a.e. $x \in \Omega$, and therefore $W^{\mathcal{\varepsilon}}$ is $L$-superharmonic.

We are now in a position to prove our main result.

Theorem 5. Let $\Omega$ be a bounded domain in $\mathbb{R}^{n}, n>2$, with the $C^{1,1}$-regularity property and let $L$ be the operator defined in (19) satisfying (20), (21), and (23). Let $n / l<p<n / 2,1<$ $v<n /(n-l+n / p)$. If $u$ is a solution of problem (18) with datum $f \in L_{v, p}^{n / v-n / p}(\Omega)$, then there exists a positive constant $C=C(n, p, l, \mu, v)$ such that

$$
\|u\|_{L^{n p /(n-2 p)}(\Omega)} \leq C\|\bar{f}\|_{L_{\nu, p}^{n / v-n / p}\left(\mathbb{R}^{n}\right)},
$$

with $\bar{f}$ as in Definition 1 .

Proof. Theorem 5 will be proved in three steps. In the first two we show some potential type estimates for the solutions of certain auxiliary problems. In the last one, we exploit the estimate of Step 2 to achieve (29).

Step 1. The datum $f \in L_{v, p}^{n / v-n / p}(\Omega)$; thus by Definition 1 there exists a function $\bar{f} \in L_{\nu, p}^{n / v-n / p}\left(\mathbb{R}^{n}\right)$ such that $\bar{f}=f$ in $\Omega$. Therefore, by (14) we can find $g \in L^{v}\left(\mathbb{R}^{n}\right)$ such that

$$
\bar{f}(x)=\int_{\mathbb{R}^{n}} \frac{g(y)}{|x-y|^{n-(n / v-n / p)}} d y, \quad x \in \mathbb{R}^{n} .
$$

Denoted by $g^{+}$and $g^{-}$the positive and negative parts of the function $g$, respectively, by classical results we get the fact that there exist two sequences of smooth and nonnegative functions $g_{h}^{+}$and $g_{h}^{-}$such that

$$
g_{h}^{ \pm} \longrightarrow g^{ \pm} \quad \text { in } L^{\nu}\left(\mathbb{R}^{n}\right) \text { as } h \longrightarrow 0 .
$$

Let $\varepsilon$ take its values in a sequence of positive reals numbers converging to zero and set

$$
\bar{f}_{h, \varepsilon}^{ \pm}(x)=\int_{\mathbb{R}^{n}} \frac{g_{h}^{ \pm}(y) d y}{\left(|x-y|^{2}+\varepsilon^{2}\right)^{(s+2) / 2}}, \quad x \in \mathbb{R}^{n},
$$


with $s=n-(n / v-n / p)-2$. Then, consider the classical solutions $u_{h, \varepsilon}^{+}$and $u_{h, \varepsilon}^{-}$of the problems

$$
\begin{gathered}
L u_{h, \varepsilon}^{+}=-\bar{f}_{h, \varepsilon}^{+} \quad \text { in } \Omega, \\
u_{h, \varepsilon}^{+}=0 \quad \text { on } \partial \Omega, \\
L u_{h, \varepsilon}^{-}=-\bar{f}_{h, \varepsilon}^{-} \text {in } \Omega, \\
u_{h, \varepsilon}^{-}=0 \quad \text { on } \partial \Omega .
\end{gathered}
$$

In this step we want show that

$$
u_{h, \varepsilon}^{ \pm}(x) \leq C_{1} \int_{\mathbb{R}^{n}} \frac{\bar{f}_{h}^{ \pm}(y)}{|x-y|^{n-2}} d y \quad \text { in } \Omega,
$$

with $C_{1}=C_{1}(n, p, l, \mu, \nu)$ positive constant, where

$$
\bar{f}_{h}^{ \pm}(x)=\int_{\mathbb{R}^{n}} \frac{g_{h}^{ \pm}(y)}{|x-y|^{s+2}} d y, \quad x \in \mathbb{R}^{n} .
$$

To this aim, put

$$
W_{h, \varepsilon}^{+}(x)=\int_{\mathbb{R}^{n}} \frac{g_{h}^{+}(y)}{\left(|x-y|^{2}+\varepsilon^{2}\right)^{s / 2}} d y, \quad x \in \Omega .
$$

In view of Lemma 4 there exists a positive constant $C^{\prime}$ such that

$$
L W_{h, \varepsilon}^{+} \leq-C^{\prime} \int_{\mathbb{R}^{n}} \frac{g_{h}^{+}(y)}{\left(|x-y|^{2}+\varepsilon^{2}\right)^{(s+2) / 2}} d y=-C^{\prime} \bar{f}_{h, \mathcal{\varepsilon}}^{+},
$$

with $C^{\prime}=C^{\prime}(n, p, l, \mu, \nu)$. Then

$$
L\left(u_{h, \varepsilon}^{+}-\frac{W_{h, \varepsilon}^{+}}{C^{\prime}}\right) \geq 0 \quad \text { in } \Omega .
$$

Moreover, by (33) and (37), one gets

$$
u_{h, \varepsilon}^{+}-\frac{W_{h, \varepsilon}^{+}}{C^{\prime}} \leq 0 \quad \text { on } \partial \Omega .
$$

Thus, as a consequence of our hypotheses, the maximum principle in its classical forms applies (see, e.g., [3], Theorems 3.1 and 3.3) and therefore by (32) and (37) we have

$$
0 \leq u_{h, \varepsilon}^{+}(x) \leq \frac{W_{h, \varepsilon}^{+}(x)}{C^{\prime}} \leq \frac{1}{C^{\prime}} \int_{\mathbb{R}^{n}} \frac{g_{h}^{+}(y)}{|x-y|^{s}} d y \quad \text { in } \Omega .
$$

This last inequality and the composition formula for fractional integrals (see, e.g., [6]) give then

$$
u_{h, \varepsilon}^{+}(x) \leq C_{1} \int_{\mathbb{R}^{n}} \frac{1}{|x-y|^{n-2}}\left(\int_{\mathbb{R}^{n}} \frac{g_{h}^{+}(z)}{|y-z|^{s+2}} d z\right) d y
$$

with $C_{1}=C_{1}(n, p, l, \mu, \nu)$ positive constant. Following the same argument one obtains a similar estimate for the function $u_{h, \varepsilon}^{-}$:

$$
u_{h, \varepsilon}^{-}(x) \leq C_{1} \int_{\mathbb{R}^{n}} \frac{1}{|x-y|^{n-2}}\left(\int_{\mathbb{R}^{n}} \frac{g_{h}^{-}(z)}{|y-z|^{s+2}} d z\right) d y
$$

in $\Omega$.

Combining inequalities (42) and (43) and definitions (36) we get (35).

Step 2. Let $u_{h, \varepsilon}$ be the classical solution of the problem

$$
\begin{gathered}
L u_{h, \varepsilon}=-\bar{f}_{h, \varepsilon} \quad \text { in } \Omega, \\
u_{h, \varepsilon}=0 \quad \text { on } \partial \Omega,
\end{gathered}
$$

where $\bar{f}_{h, \varepsilon}=\bar{f}_{h, \varepsilon}^{+}-\bar{f}_{h, \varepsilon}^{-}$.

In view of the uniqueness of the solutions of problems (33), (34), and (44) (see, e.g., [3], Theorem 9.15) one has that $u_{h, \varepsilon}=u_{h, \varepsilon}^{+}-u_{h, \varepsilon}^{-}$and, therefore, since $u_{h, \varepsilon}^{ \pm}$are nonnegative, as observed in (41), $\left|u_{h, \varepsilon}\right| \leq u_{h, \varepsilon}^{+}+u_{h, \varepsilon}^{-}$. Thus, (35) gives the following potential type estimate for $u_{h, \varepsilon}$ :

$$
\left|u_{h, \varepsilon}(x)\right| \leq C_{1} \int_{\mathbb{R}^{n}} \frac{\left[\bar{f}_{h}^{+}(y)+\bar{f}_{h}^{-}(y)\right]}{|x-y|^{n-2}} d y \quad \text { in } \Omega .
$$

We want to pass to the limit as $\varepsilon \rightarrow 0$ in (45).

By classical estimates (observe that the coefficients of problem (44) are smooth and, therefore [3], Lemma 9.17 applies) we get

$$
\left\|u_{h, \varepsilon}-u_{h, \varepsilon^{\prime}}\right\|_{W^{2, p}(\Omega)} \leq C^{\prime \prime}\left\|\bar{f}_{h, \varepsilon}-\bar{f}_{h, \varepsilon^{\prime}}\right\|_{L^{p}(\Omega)} .
$$

For $\bar{f}_{h, \varepsilon}^{+}$and $\bar{f}_{h, \varepsilon}^{-}$, for any fixed $h$, by (36), (32), and Beppo Levi's theorem, we obtain

$$
\bar{f}_{h, \varepsilon}^{ \pm}(x) \longrightarrow \bar{f}_{h}^{ \pm}(x) \quad \text { a.e. in } \mathbb{R}^{n} \text { as } \varepsilon \longrightarrow 0 .
$$

By Lebesgue's dominated convergence theorem, these last convergences take place in $L^{p}\left(\mathbb{R}^{n}\right)$ too:

$$
\bar{f}_{h, \varepsilon}^{ \pm}(x) \longrightarrow \bar{f}_{h}^{ \pm}(x) \quad \text { in } L^{p}\left(\mathbb{R}^{n}\right) \text { as } \varepsilon \longrightarrow 0,
$$

and thus

$$
\bar{f}_{h, \varepsilon}(x) \longrightarrow \bar{f}_{h}(x) \quad \text { in } L^{p}\left(\mathbb{R}^{n}\right) \text { as } \varepsilon \longrightarrow 0,
$$

where we set

$$
\bar{f}_{h}=\bar{f}_{h}^{+}-\bar{f}_{h}^{-} .
$$

Putting together (46) and (49), we derive that $u_{h, \varepsilon}$ is a Cauchy sequence in $W^{2, p}(\Omega)$. Thus, by the completeness of $W^{2, p}(\Omega)$, there exists $u_{h} \in W^{2, p}(\Omega)$ such that $u_{h, \varepsilon} \rightarrow u_{h}$ in $W^{2, p}(\Omega)$, as $\varepsilon \rightarrow 0$. Hence, up to a subsequence, still denoted by $u_{h, \varepsilon}$, we have

$$
u_{h, \varepsilon} \longrightarrow u_{h} \quad \text { a.e. in } \Omega \text { as } \varepsilon \longrightarrow 0 \text {. }
$$


Taking into account (51), we can finally pass to the limit as $\varepsilon \rightarrow 0$ in (45), obtaining the following potential type estimate for $u_{h}$ :

$$
\left|u_{h}(x)\right| \leq C_{1} \int_{\mathbb{R}^{n}} \frac{\left[\bar{f}_{h}^{+}(y)+\bar{f}_{h}^{-}(y)\right]}{|x-y|^{n-2}} d y \quad \text { in } \Omega .
$$

Step 3. Combining (52) and (13) we have

$$
\begin{aligned}
& \left\|u_{h}\right\|_{L^{n p /(n-2 p)}(\Omega)} \\
& \leq C_{1}\left(\int_{\Omega}\left(\int_{\mathbb{R}^{n}} \frac{\left[\bar{f}_{h}^{+}(y)+\bar{f}_{h}^{-}(y)\right]}{|x-y|^{n-2}} d y\right)^{n p /(n-2 p)} d x\right)^{(n-2 p) / n p} \\
& \leq C_{1}\left\|I^{2}\left(\bar{f}_{h}^{+}+\bar{f}_{h}^{-}\right)\right\|_{L^{n p /(n-2 p)\left(\mathbb{R}^{n}\right)}} \leq C_{2}\left\|\bar{f}_{h}^{+}+\bar{f}_{h}^{-}\right\|_{L^{p}\left(\mathbb{R}^{n}\right)} \\
& \leq C_{2}\left(\left\|\bar{f}_{h}^{+}\right\|_{L^{p}\left(\mathbb{R}^{n}\right)}+\left\|\bar{f}_{h}^{-}\right\|_{L^{p}\left(\mathbb{R}^{n}\right)}\right),
\end{aligned}
$$

with $C_{2}=C_{2}(n, p, l, \mu, v)$.

To prove our claim, we want to pass to the limit as $h \rightarrow 0$ in (53).

From (31), (36), and (13), it follows that

$$
\bar{f}_{h}^{ \pm}(x) \longrightarrow \bar{f}^{ \pm}(x) \quad \text { in } L^{p}\left(\mathbb{R}^{n}\right) \text { as } h \longrightarrow 0,
$$

where we set

$$
\begin{aligned}
& \bar{f}^{+}(x)=\int_{\mathbb{R}^{n}} \frac{g^{+}(y)}{|x-y|^{n-(n / v-n / p)}} d y, \\
& \bar{f}^{-}(x)=\int_{\mathbb{R}^{n}} \frac{g^{-}(y)}{|x-y|^{n-(n / v-n / p)}} d y,
\end{aligned}
$$

$x \in \mathbb{R}^{n}$

Thus, since $\bar{f}=\bar{f}^{+}-\bar{f}^{-}$, by (54) we have

$$
\bar{f}_{h}(x) \longrightarrow \bar{f}(x) \quad \text { in } L^{p}\left(\mathbb{R}^{n}\right) \text { as } h \longrightarrow 0 .
$$

Now, observe that, by uniqueness, $u_{h}$ is the solution of the problem

$$
\begin{gathered}
L u_{h}=-\bar{f}_{h} \quad \text { in } \Omega, \\
u_{h}=0 \quad \text { on } \partial \Omega .
\end{gathered}
$$

Classical results give then

$$
\left\|u_{h}-u_{h^{\prime}}\right\|_{W^{2, p}(\Omega)} \leq C^{\prime \prime \prime}\left\|\bar{f}_{h}-\bar{f}_{h^{\prime}}\right\|_{L^{p}(\Omega)},
$$

and so, in view of convergence (56), we obtain

$$
u_{h} \longrightarrow u \text { in } W^{2, p}(\Omega), \text { as } h \longrightarrow 0,
$$

where $u$ is the solution of problem (18). that

The embedding $W^{2, p}(\Omega) \subset L^{n p /(n-2 p)}(\Omega)$ then ensures

$$
u_{h} \longrightarrow u \quad \text { in } L^{n p /(n-2 p)}(\Omega), \text { as } h \longrightarrow 0 .
$$

Finally, by convergences (54) and (60) we can pass to the limit as $h \rightarrow 0$ in (53), obtaining

$$
\|u\|_{L^{n p /(n-2 p)}(\Omega)} \leq C_{2}\left(\left\|\bar{f}^{+}\right\|_{L^{p}\left(\mathbb{R}^{n}\right)}+\left\|\bar{f}^{-}\right\|_{L^{p}\left(\mathbb{R}^{n}\right)}\right) .
$$

We are now able to conclude our proof. Indeed, by (61) and (13) we get

$$
\|u\|_{L^{n p /(n-2 p)}(\Omega)} \leq C_{3}\left(\left\|g^{+}\right\|_{L^{\nu}\left(\mathbb{R}^{n}\right)}+\left\|g^{-}\right\|_{L^{\nu}\left(\mathbb{R}^{n}\right)}\right),
$$

with $C_{3}=C_{3}(n, p, l, \mu, \nu)$.

Moreover,

$$
\|g\|_{L^{y}\left(\mathbb{R}^{n}\right)} \approx\left\|g^{+}\right\|_{L^{y}\left(\mathbb{R}^{n}\right)}+\left\|g^{-}\right\|_{L^{y}\left(\mathbb{R}^{n}\right)},
$$

the functions $g^{+}$and $g^{-}$having disjoint supports.

Thus, by (62), (63), and (16), we obtain our estimate

$$
\begin{aligned}
\|u\|_{L^{n p /(n-2 p)}(\Omega)} & \leq C_{4}\|g\|_{L^{\nu}\left(\mathbb{R}^{n}\right)}=C_{4}\left\|\mathbb{D}^{n / v-n / p} f\right\|_{L^{\nu}\left(\mathbb{R}^{n}\right)} \\
& \leq C_{4}\|\bar{f}\|_{L_{\nu, p}^{n / v-n / p}\left(\mathbb{R}^{n}\right)},
\end{aligned}
$$

with $C_{4}=C_{4}(n, p, l, \mu, \nu)$.

\section{Disclosure}

Sara Monsurrò and Maria Transirico are Members of the Gruppo Nazionale per l'Analisi Matematica, la Probabilità e le loro Applicazioni (GNAMPA) of the Istituto Nazionale di Alta Matematica (INdAM).

\section{Conflict of Interests}

The authors declare that there is no conflict of interests regarding the publication of this paper.

\section{References}

[1] G. Talenti, "Sopra una classe di equazioni ellittiche a coefficienti misurabili," Annali di Matematica Pura ed Applicata, vol. 69, pp. 285-304, 1965.

[2] A. Maugeri, D. K. Palagachev, and L. G. Softova, Elliptic and Parabolic Equations with Discontinuous Coefficients, vol. 109 of Mathematical Research, Wiley-VCH, Berlin, Germany, 2000.

[3] D. Gilbarg and N. S. Trudinger, Elliptic Partial Differential Equations of Second Order, Classics in Mathematics, Springer, Berlin, Germany, 2001.

[4] S. Campanato, "Un risultato relativo ad equazioni ellittiche del secondo ordine di tipo non variazionale," Annali della Scuola Normale Superiore di Pisa, vol. 21, pp. 701-707, 1967.

[5] F. Mamedov, S. Monsurró, and M. Transirico, "Potential estimates and applications to elliptic equations," Discrete and Continuous Dynamical Systems Supplement, vol. 2015, pp. 793800, 2015.

[6] N. S. Landkof, Foundations of Modern Potential Theory, vol. 180 of Die Grundlehren der Mathematischen Wissenschaften, Springer, New York, NY, USA, 1972.

[7] S. G. Samko, Hypersingular Integrals and Their Applications (Analytical Methods and Special Functions), Taylor \& Francis, London, UK, 2002. 
[8] S. G. Samko, A. A. Kilbas, and O. I. Marichev, Fractional Integrals and Derivatives. Theory and Applications, Gordon and Breach Science Publishers, Yverdon, Switzerland, 1993, Translated from the 1987 Russian Original.

[9] E. M. Stein, Singular Integrals and Differentiability Properties of Functions, Princeton Mathematical Series no. 30, Princeton University Press, Princeton, NJ, USA, 1970. 


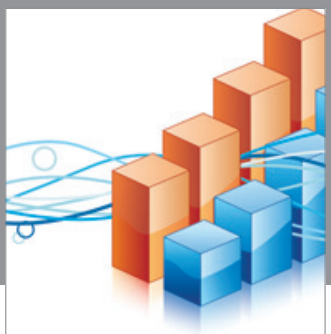

Advances in

Operations Research

vatem alat4

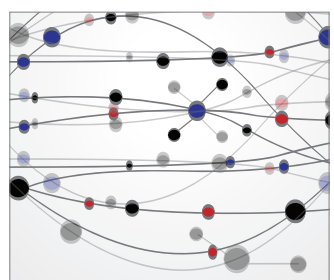

\section{The Scientific} World Journal
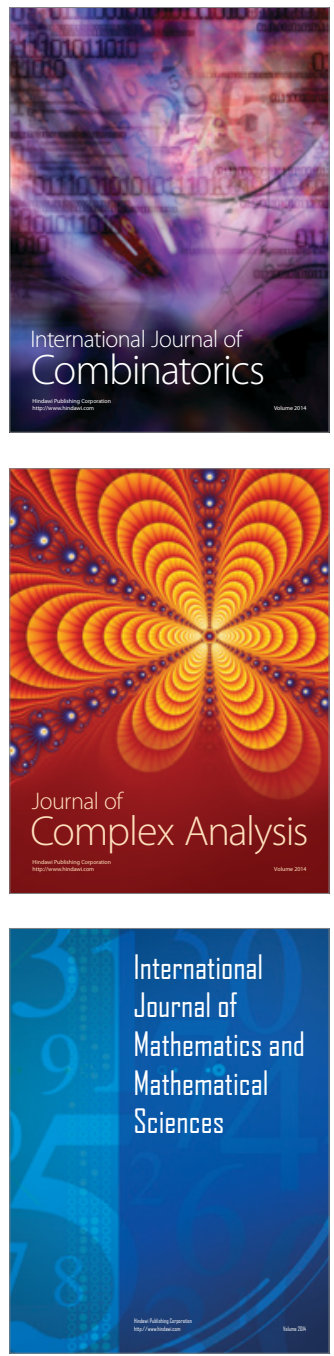
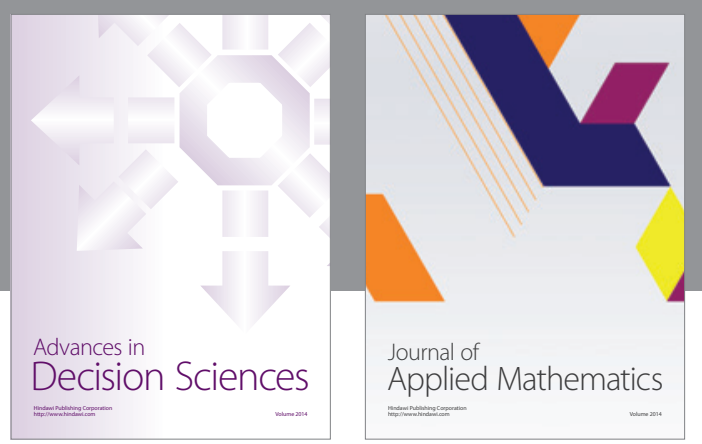

Algebra

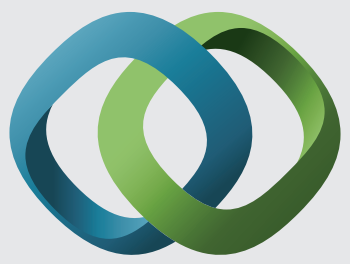

\section{Hindawi}

Submit your manuscripts at

http://www.hindawi.com
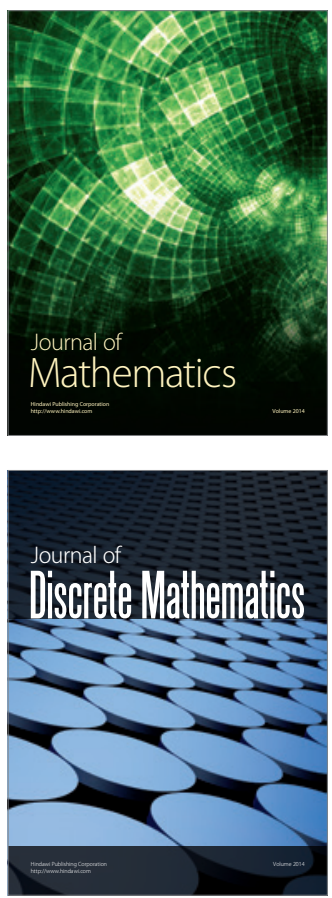

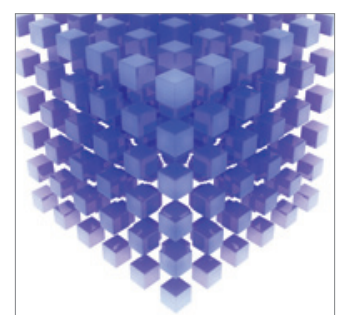

Mathematical Problems in Engineering
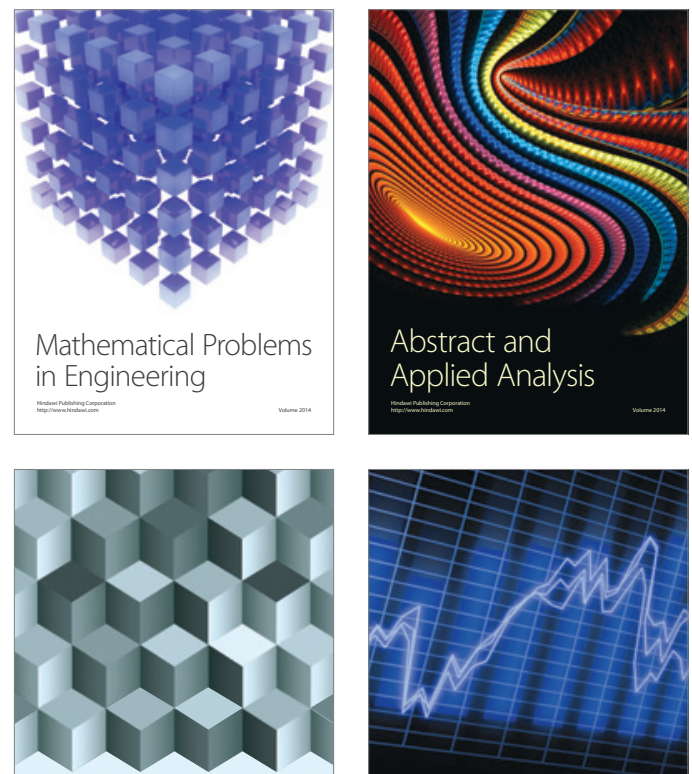

Journal of

Function Spaces

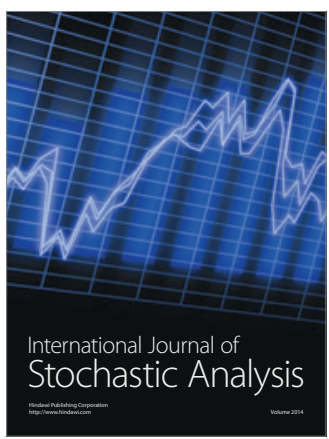

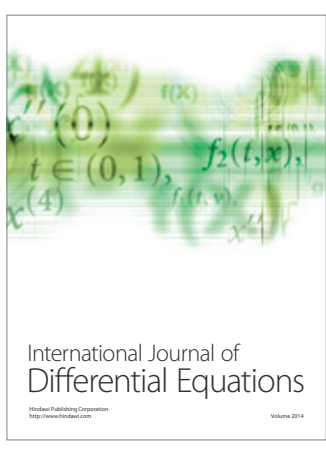
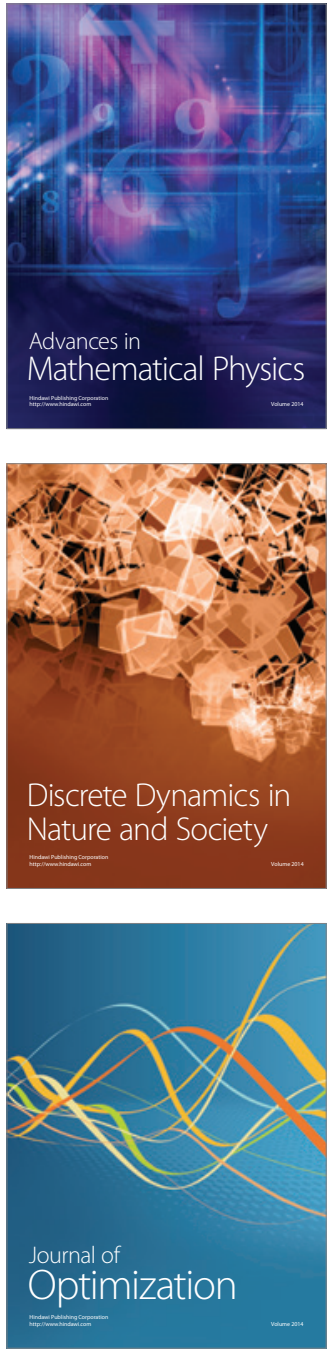